Revue musicale OICRM

\title{
Le concert participatif. Une forme efficiente pour partager la médiation de la musique classique
}

\section{Cécile Prévost-Thomas}

Volume 7, numéro 2, 2020

Fenêtre ouverte sur la médiation de la musique

URI : https://id.erudit.org/iderudit/1072414ar

DOI : https://doi.org/10.7202/1072414ar

Aller au sommaire du numéro

Éditeur(s)

Observatoire interdisciplinaire de création et recherche en musique (OICRM)

ISSN

2368-7061 (numérique)

Découvrir la revue

Citer cet article

Prévost-Thomas, C. (2020). Le concert participatif. Une forme efficiente pour partager la médiation de la musique classique. Revue musicale OICRM, 7(2), 36-52. https://doi.org/10.7202/1072414ar
Résumé de l'article

À partir de l'observation de La nuit des Mayas, concert participatif issu du dispositif de concerts-médiation Musiques entre les lignes, conçu par Thierry Weber à la Haute École de Musique de Lausanne, cet article propose de mesurer les différentes dimensions, effets et enjeux du concept de participation à travers son application au secteur de la médiation de la musique. 


\title{
Le concert participatif. Une forme efficiente pour partager la médiation de la musique classique
}

\author{
Cécile Prévost-Thomas
}

\begin{abstract}
Résumé
À partir de l'observation de La nuit des Mayas, concert participatif issu $\mathrm{du}$ dispositif de concerts-médiation Musiques entre les lignes, conçu par Thierry Weber à la Haute École de Musique de Lausanne, cet article propose de mesurer les différentes dimensions, effets et enjeux du concept de participation à travers son application au secteur de la médiation de la musique.

Mots clés : concert-médiation ; jeune public ; médiation de la musique ; musicienmédiateur ; participation.

Abstract

From the observation of La nuit des Mayas, a participatory concert based on the concert-mediation plan Musiques entre les lignes, designed by Thierry Weber at the Haute École de Musique of Lausanne, this article aims at measuring the different dimensions, effects and issues of the concept of participation through its application to the music mediation sector.
\end{abstract}

Keywords: mediation-concert; music mediation; musician-mediator; participation; young audience. 
C'est en mettant en œuvre et en action, et par conséquent en supposant une totale participation, que l'appropriation peut véritablement se faire. (Chaumier 2016, p. 227)

Parallèlement aux enseignements dédiés à la médiation de la musique que je dispense à la Sorbonne depuis plus de dix ans ${ }^{1}$, aux commandes d'enquêtes de terrain qui m'ont été confiées par des institutions musicales françaises ${ }^{2}$ et à l'événement scientifique "Les mondes de la médiation culturelle », que j'ai coorganisé sur le sujet en 2013 à la Sorbonne Nouvelle ${ }^{3}$, j'ai eu récemment l'opportunité d'enseigner la médiation de la musique et de poursuivre mes recherches dans ce domaine auprès de la Haute École de Musique de Lausanne (HEMU) et de l'Institut de recherche en Musique et Arts de la scène (IRMAS). Durant ces deux années, j'ai donc profité du terrain helvète pour affiner mes réflexions à la fois sur le plan théorique et sur le plan empirique en observant de près et sur une longue durée le dispositif Musique entre les lignes.

Si ma principale recherche, Partager la musique. Des musiciens à la rencontre des publics $^{4}$, consacrée à ce programme s'est déroulée sur l'année académique 2018-2019, j'ai bénéficié d'une première année, entre 2017 et 2018, pour m’immerger sur le terrain des actions de médiation proposées par l'HEMu en enseignant, entre autres à certain $\cdot e \cdot s$ étudiant $\cdot e \cdot s$ de cette école, la méthodologie de l'observation appliquée au terrain spécifique des concerts-médiation proposés par Musique entre les lignes. Au-delà de l'accompagnement pédagogique et de la transmission de ces connaissances et compétences spécifiques, rarement enseignées aux musicien·ne·s, en plus de l'immersion collective sur le terrain de la médiation musicale que ces nouveaux savoir-faire nous permettaient de vivre en commun, en amont et en aval des concerts, l'objectif des séances d'observation était aussi de comprendre le sens qui était donné aux différentes formes de médiation choisies par l'équipe de production du dispositif, alternant selon le programme musical retenu, des concerts expliqués ou participatifs.

1 J'ai d'abord dispensé un enseignement de «Sociologie des publics de la musique » au sein du Master 2 Recherche Musique et musicologie, option Médiation musicale entre 2008 et 2010 à 1'Université ParisSorbonne (Paris IV), puis j'ai mis sur pied à la rentrée 2014 le Master Médiation de la Musique à la Sorbonne Nouvelle (Département de Médiation culturelle) avec Hyacinthe Ravet pour Sorbonne Université (UFR de Musique et musicologie). Depuis la création de cette formation, je dispense plusieurs cours théoriques, pratiques et méthodologiques et j'encadre de nombreux travaux de recherche dédiés spécifiquement à la médiation de la musique en Master 1 et 2.

2 Évaluations de dispositifs de médiation de la musique auprès de l'Orchestre français des jeunes (2012-2013), de la Philharmonie de Paris (2014-2016), des Talens Lyriques (2014-2018).

3 J'ai été, avec plusieurs de mes collègues du département de médiation culturelle de la Sorbonne Nouvelle, à l'initiative du colloque «Les mondes de la médiation culturelle», qui s'est tenu à Paris du 17 au 19 octobre 2013. Plusieurs interventions spécifiquement dédiées à la musique ont été proposées à l'occasion de cet événement. Une partie d'entre elle sont disponibles dans le second volume de la publication des actes du colloque (voir Camart et al. 2016).

4 Cette recherche dont la méthodologie était à la fois quantitative (questionnaire destiné aux publics) et qualitative (entretiens avec l'équipe du dispositif et avec les parents et enfants) a été cofinancé par l'HEMU et l'IRMAS. 
La réflexion proposée ici se focalisera particulièrement sur le concert dit participatif, cette catégorie semblant non seulement répondre de manière précise aux différents enjeux (sociaux, culturels, esthétiques) que pose la médiation de la musique, mais aussi produire des effets tangibles pour rendre à la fois la musique classique plus accessible et pour partager pleinement l'expérience même de la médiation.

\section{MUSIQUE ENTRE LES LIGNES : UN ESPRIT PARTICIPATIF}

En 2017-2018, pour la quatrième année consécutive, le programme Musique entre les lignes impulsé au sein de l'HEMu par le chef d'orchestre, pédagogue et directeur artistique Thierry Weber depuis 2014, poursuit le développement de ses concertsmédiation en direction des publics scolaires et des familles. Avec le temps, chaque concert, créé au cœur del'institution, dans le Bvс Concert Hall et présenté parl'ensemble instrumental de l'HEMU composé d'étudiant·e·s en formation sous la direction de Weber, se démultiplie en représentations extérieures dédiées aux publics des cantons du Valais et de Fribourg. D'année en année, le dispositif de ses concerts-médiation s'enrichit de nouvelles formes de médiation, alternant des concerts expliqués, des contes musicaux, des concerts participatifs, etc. L'association d'artistes divers (comédien·ne·s, marionnettistes, circassien circassien·ne·s) intervenant régulièrement dans ce dispositif encourage par ailleurs une dynamique interartistique propice aux activités de médiation de la musique, comme nous le verrons plus loin.

L'ambition de Musique entre les lignes est donc double : transmettre d'une part, sous forme de concerts-médiation, "la musique classique dans des lieux et auprès de publics desquels elle semble exclue, considérant que l'éducation artistique est un enjeu politique, pédagogique et culturel majeur » et, d'autre part " éveiller les musiciens, futurs professionnels, aux enjeux pédagogiques de demain, tout en leur donnant de réelles opportunités de pratiques " (HEMU 2016, p. 8). Mais plus encore, l'objectif de ce dispositif de médiation musicale est de permettre aux uns, les publics, et aux autres, les musicien·ne·s et les artistes extérieur·e·s invité·e·s, de vivre l'expérience du concert, le moment de la rencontre musicale comme un temps commun privilégié suscitant des échanges (verbaux, corporels, musicaux, vocaux) à partir et autour des œuvres inscrites au programme.

Pour créer du lien entre les œuvres et les publics, principe au fondement même de la médiation, de sa raison d'être et de son utilité sociale, une des caractéristiques de ce dispositif est de recourir à l'intervention de médiateurs'rice-s-artistes comme le·la chef·fe d'orchestre, les musicien·ne·s ou les artistes associé·e·s : comédien·ne, marionnettiste, etc. Cette précision est importante puisqu'elle permet de penser la spécificité de la fonction de l'artiste-médiateur-rice qui doit pouvoir conjuguer son statut d'artiste avec sa capacité à " produire des outils pédagogiques, de communication, de dialogue, trouver les bonnes manières pour véhiculer des contenus avec sensibilité pour que les participants se les approprient » (Kaine, Bergeron-Martel et Morasse 2017, p. 331).

Initiateur de ce projet, Weber en est le chef d'orchestre à double titre, celui de sa conception artistique adaptée à une forme de médiation spécifique, et celui qui accompagne les musicien·ne·s en formation à appréhender et à réaliser une forme de 
concert où la musique qu'ils elles interprètent tient une place aussi importante que la relation qu'elle permet d'entretenir avec le public présent lors de la performance offerte et partagée.

Qu'il soit « scolaire » ou « familial », le public auquel s'adresse le programme, toujours composé d'enfants, est donc pensé en amont, dès les prémices du processus de création ; par ailleurs, ce dernier n'est pas, pour reprendre l'expression de Serge Chaumier (2016, p. 232), " une cible », mais « un centre » qui " justifie l'existence » de Musiques entre les lignes. Autrement dit, quelle que soit la forme de médiation adoptée (concert expliqué, concert participatif, conte musical), " sans jamais négliger la qualité et l'excellence artistique, chaque projet est conçu dans un esprit participatif favorisant l'appropriation des auditeurs » (HEMU 2017b, p. 6) excluant ainsi la figure du consommateur culturel passif.

\section{LA NUIT DES MAYAS : OBSERVATION AU CEUR D'UN CONCERT PARTICIPATIF}

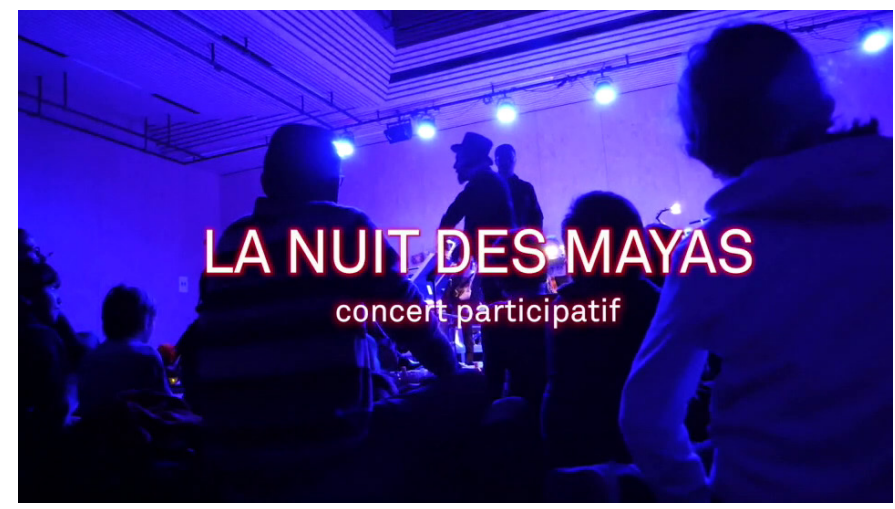

Figure 1 : Titre du documentaire La nuit des Mayas ${ }^{5}$.

Le dispositif de type participatif est donc une des formes plébiscitées par Musiques entre les lignes pour faire acte et œuvre de médiation depuis la saison 2016-20176. Appartenant à cette catégorie, "La nuit des Mayas ", concert-médiation conçu par Thierry Weber et créé les 7, 8 et 9 novembre 2017 et dont nous avons observé en direct les trois séances selon la technique de l'observation non-participante à partir d'une grille spécifique ${ }^{7}$, servira de modèle à notre démonstration. Inspiré par La Noche

5 Les photos reproduites dans cet article sont des captures d'images effectuées à partir de la captation vidéo de Jean-Marc Bordet, réalisée pour Musiques entre les lignes et disponible à la fin de cet article.

6 «Le premier concert de l'Ours Paddington » (décembre 2016), « Les cygnes sauvages » (mai 2017) étaient présentés comme des " contes musicaux participatifs ».

$7 \quad$ Cette grille que nous avons également mise à l'épreuve d'une autre série de concerts expliqués, «Jazz'Noizet », avec les étudiant·e·s de l'HEMu en décembre 2017 proposait les indicateurs suivants : heure, durée, lieu (description), plan, espace (utilisation, postures, répartition, déambulation, etc.), participant·e·s (nombre, nature, identification), actions, activités (répartition parole, jeu, écoute), outils de médiation (instruments pour jouer l'œuvre ou comme soutien aux actions de médiation, autres supports, autres expressions artistiques), action des médiateur rice's (présentation, discours, mode d'adresse, vocabulaire, occupation de l'espace, rapport scène/salle, répartition des rôles, de la prise de parole, etc.), réactions du public (attitudes verbales, non verbales/ langage du corps, relations enfants/adultes, évolution des comportements entre le début et la fin du concert.), interactions (médiateur·rice/enfants ; médiateur-rice/adultes ; enfants/enfants, etc.). 
de los Mayas (1939), œuvre contemporaine pour orchestre de Silvestre Revueltas ${ }^{8}$, cette création collective (un chef d'orchestre, une quinzaine de musicien·ne.s et un comédien) a permis de mettre en relief les différentes possibilités de médiation qu'offre ce type de concert, la notion de participation ayant été pour l'occasion envisagée selon différentes perspectives.

Afin de les saisir dans leur diversité et leur complémentarité pour comprendre quels effets elles peuvent avoir sur leurs destinataires (publics et musicien·ne·s), un retour sur le concept de participation et sur les pratiques qui en découlent dans le milieu du spectacle vivant et au-delà servira de préambule.

\section{PARTicipation CUlTURELle, PARTICIPATION CITOYENNE ET VIVRE-ENSEMBLE}

Si la participation des publics en tant qu'acteurs mobilisés au cœur des représentations artistiques (théâtrales, musicales) remonte à la Grèce antique (Siohan 1967), on retiendra brièvement pour l'époque contemporaine un intérêt croissant pour ce type d'activité à partir des années 1960 avec l'élan conjoint des mouvements d'éducation populaire, des pédagogies innovantes et de l'influence de figures emblématiques comme Augusto Boal et son Théâtre de l'Opprimé au sein duquel les spectateurs peuvent devenir des acteurs pour « se libérer de leur condition, agir et penser par eux-mêmes » (Boal [1977]1996, p. 48).

Si l'on admet dans cette lignée que la participation est « un processus par lequel un individu ou un groupe acquiert les moyens de renforcer sa capacité d'action, de s'émanciper » (Bacqué, Rey et Sintomer 2005, cités par Pailler 2018, p. 61), toute expérience sociale qui fait appel à la participation convoque le champ du politique puisqu'au-delà d'une participation artistique ou culturelle germe l'idée d'une participation citoyenne. C'est d'ailleurs à partir de ce point d'ancrage que la notion de participation s'est peu à peu redéployée puis imposée ces dix dernières années au sein des institutions culturelles (musées, lieux de concerts, bibliothèques, etc.), faisant ainsi écho au mouvement plus large de démocratie participative en vogue, du moins en France, au tournant des années 2000. Dans ce sens, « la participation s'adresse à la pensée des citoyens. Cela n'a rien d'un cri, c'est une réflexion sur les propositions qui sont faites aux citoyens pour améliorer leur vie collective, leurs rapports entre eux, mais aussi leurs rapports à la culture, à l'économie, à l'organisation générale de la vie » (Ladsous 2017, p. 209).

Enfin, l'engouement récent pour les formes participatives dans le secteur culturel répond à la volonté politique du "droit [de chacun] à participer à la vie culturelle » (Pailler 2018, p. 61) comme le précise l'article 5 (accès et participation à la vie culturelle) de la Déclaration de Fribourg sur les droits culturels qui dit :

a. Toute personne, aussi bien seule qu'en commun, a le droit d'accéder et de participer librement, sans considération de frontières, à la vie culturelle à travers les activités de son choix. 
b. Ce droit comprend notamment :

- la liberté de s'exprimer, en public ou en privé dans la, ou les, langues de son choix ;

- la liberté d'exercer, en accord avec les droits reconnus dans la présente Déclaration, ses propres pratiques culturelles et de poursuivre un mode de vie associé à la valorisation de ses ressources culturelles, notamment dans le domaine de l'utilisation, de la production et de la diffusion de biens et de services ;

- la liberté de développer et de partager des connaissances, des expressions culturelles, de conduire des recherches et de participer aux différentes formes de création ainsi qu'à leurs bienfaits ;

- le droit à la protection des intérêts moraux et matériels liés aux œuvres qui sont le fruit de son activité culturelle. (Les droits culturels, p. 6-7)

De manière encore plus explicite, on partagera avec Danielle Pailler reprenant les dires de Christian Poirier, chercheur à l'Observatoire des médiations culturelles de Montréal (Pailler 2018), l'idée selon laquelle la « participation considérée dans le contexte de la citoyenneté culturelle » correspond à l'

ensemble des activités artistiques et culturelles réalisées par des individus et des groupes sur les plans de la création, de la production, de la diffusion et de la fréquentation, et ce selon une perspective centrée sur la démocratie et la citoyenneté culturelles, sur les relations entre les citoyens, le tissu associatif et les institutions, ainsi que sur les impacts élargis de la culture pour les individus, les communautés et le vivre-ensemble (Poirier 2016, cité par ibid., p. 62)

À l'aune de ces deux conceptions, celle de la participation qui active la médiation et celle de la participation qui justifie le vivre-ensemble (Caune 2017, Lamoureux et Uhl 2018), il semble judicieux de comprendre quels différents sens revêt la participation dans un environnement artistique comme celui du concert participatif proposé par l'HEMU. Il est important cependant de considérer le cas singulier de cette proposition de médiation de la musique qui. rappelons-le, est destinée à un jeune public, qui plus est, encadré et accompagné par des adultes (enseignants, parents, grands-parents, etc.). Les modalités de participation en jeu doivent donc forcément s'adapter à ce type de public singulier et les enjeux qui en découlent sont spécifiques à cette catégorie. Dans ce sens, comme le souligne Nathalie Casemajor, Ėve Lamoureux et Danièle Racine : «il est essentiel de ne pas confondre, dans l'analyse des enjeux, le public adulte volontaire et le jeune public qui, bien souvent, est "captif" " (Casemajor, Lamoureux et Racine 2015, p. 174).

\section{DES ENFANTS SPECT-ACTEURS}

Pour autant dans le cas précis de «La nuit des Mayas », la proposition de médiation musicale offerte aux enfants les invite justement à "prendre part activement " au concert, au même titre que les musicien'ne·s, le chef d'orchestre, le comédien associé, partageant tous ensemble le moment collectif du concert participatif. Ainsi, la forme adoptée par ce dispositif offre la possibilité de penser différemment la notion de jeune public qui n'est plus accueilli comme simple spectateur captif mais qui devient spect- 
acteur ${ }^{9}$, puisqu'il est tantôt invité à échanger, interagir, écouter, regarder, observer, se mouvoir (figure 2), jouer de plusieurs instruments - maracas et güiros préalablement fabriqués pour l'occasion ${ }^{10}$ - (figure 3), déambuler au cœur de l'orchestre, chanter le refrain d'une chanson incluse dans le concert, danser, etc., et tantôt convié à interpréter différents rôles (chef d'orchestre, animateur-rice musical·e de son propre groupe) au fur et à mesure du développement du concert-médiation.

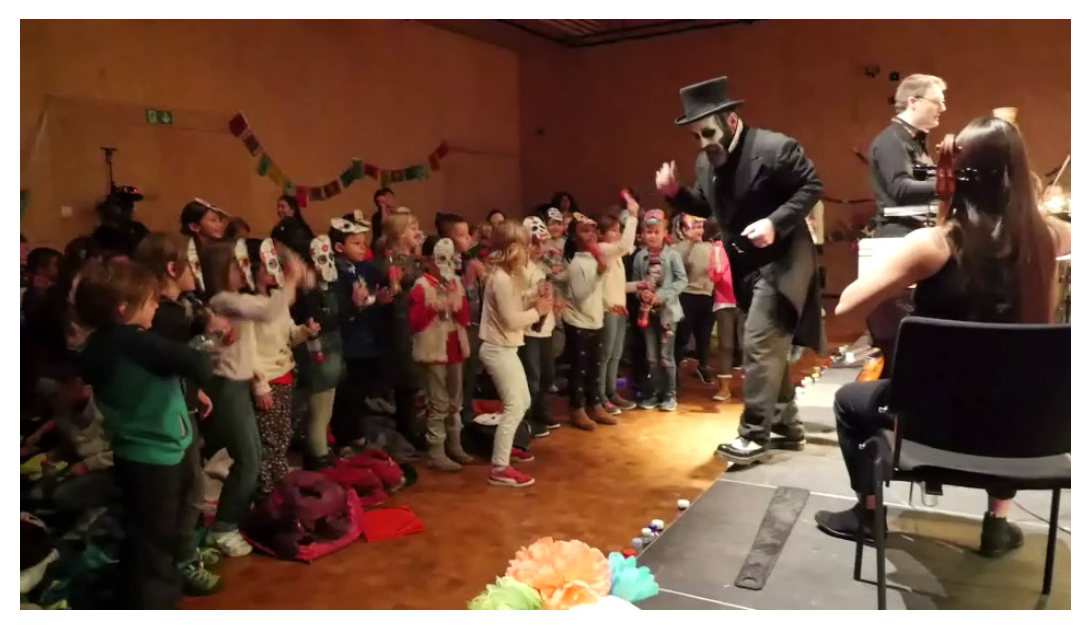

Figure 2: Des enfants spect-acteurs 1.

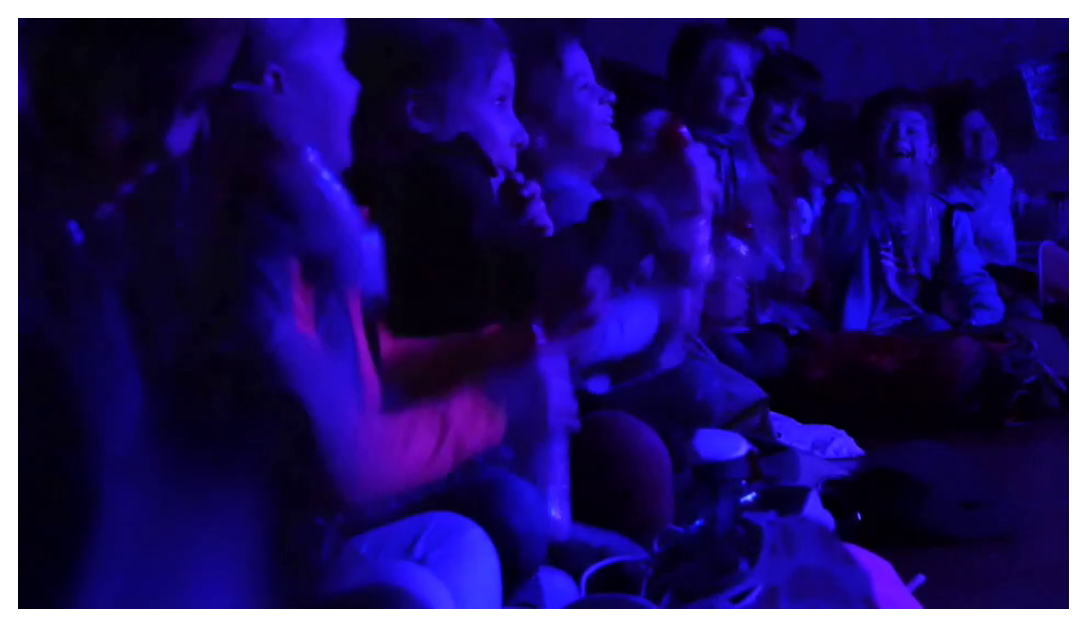

Figure 3 : Des enfants spect-acteurs 2.

Pour une même proposition artistique comme celle de «La nuit des Mayas », qui constitue une série de plusieurs représentations, la participation proposée aux enfants permet de surcroît de vivre à chaque fois une expérience unique. En prenant part à l'action du concert dès leur entrée dans la salle du Bvc Concert Hall où les accueillait le comédien qui les accompagnaient jusqu'à l'espace qui leur était réservé pour profiter

9 On doit cette expression à Augusto Boal, équivalente sans distinction à celles de spectateur-participant ou spectateur-acteur, qui désigne ainsi dans la pratique du théâtre-forum le spectateur qui devenant acteur ou joueur « intervient dans l'action et la modifie » (Boal [1977]1996, p. 32-35).

10 Cet aspect du dispositif est expliqué plus loin. 
au maximum de l'expérience, en exprimant leur spontanéité corporelle, en formulant leurs interrogations et en livrant naturellement leurs mots d'enfants, en déployant leur imaginaire guidé par le propos du comédien qui les invite à se plonger dans l'univers d'El día de los muertos ${ }^{11}$, soutenu par l'interprétation de l'ensemble instrumental de 1'HEMu à la découverte des œuvres Sensemayá de Silvestre Revueltas (créée en 1938) et Danzón n ${ }^{\circ} 2$ d'Arturo Márquez (créée en 1994) qui constituent l'ossature du concert, et stimulé par les interventions du chef d'orchestre, les enfants « interviennent dans l'action et la modifie ${ }^{12}$.

\section{DES MUSICIEN·NE·S-MÉDIATEUR·RICE·S}

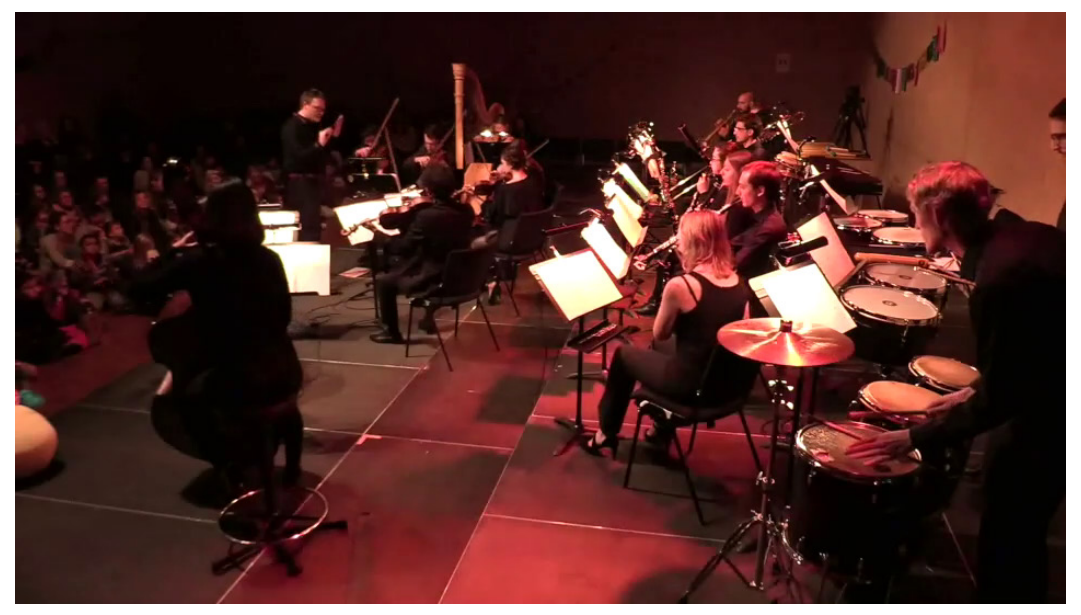

Figure 4 : Des musicien·ne·s-médiateur-rice•s en action.

Le caractère unique de chaque concert est également éprouvé par les musicien·ne's qui s'engagent dans une telle série. Même s'ils·elles sont encadré·e·s et accompagné·e·s par le chef dans chacune des étapes du processus de création en amont et qu'ils·elles sont dirigé·e·s sur scène pour interpréter les œuvres en suivant scrupuleusement leur partition, la dimension participative de chaque concert les incite à communiquer plus facilement avec leur auditoire, à adopter une posture corporelle différente, à interagir verbalement, à improviser des fragments musicaux (figure 4), etc. De plus, l'observation d'une série comme celle de «La nuit des Mayas » permet de remarquer le lâcher-prise des musicien·ne·s d'une représentation à l'autre, la progression de leur assurance et l'intensité grandissante de leur plaisir à interagir avec ce public qui devient un peu le leur (figure 5), le temps de la durée de la série de concerts.

11 El día de los muertos (le « Jour des morts ») est un rituel célébré au Mexique chaque année les $1^{\text {er }}$ et 2 novembre au cours duquel les Mexicains honorent festivement - en mangeant, dansant et chantant - leurs morts dans les cimetières. Pour l'occasion, ils leur font des offrandes et confectionnent chez eux des autels à leur mémoire composés de : fleurs porte-bonheur - œillets d'Inde -, cierges et bougies, calaveras - têtes de mort en sucre, en chocolats ou en plastique pour la décoration, encens - copal - qui symbolise le passage de la vie à la mort, etc.

12 Voir note 9. 


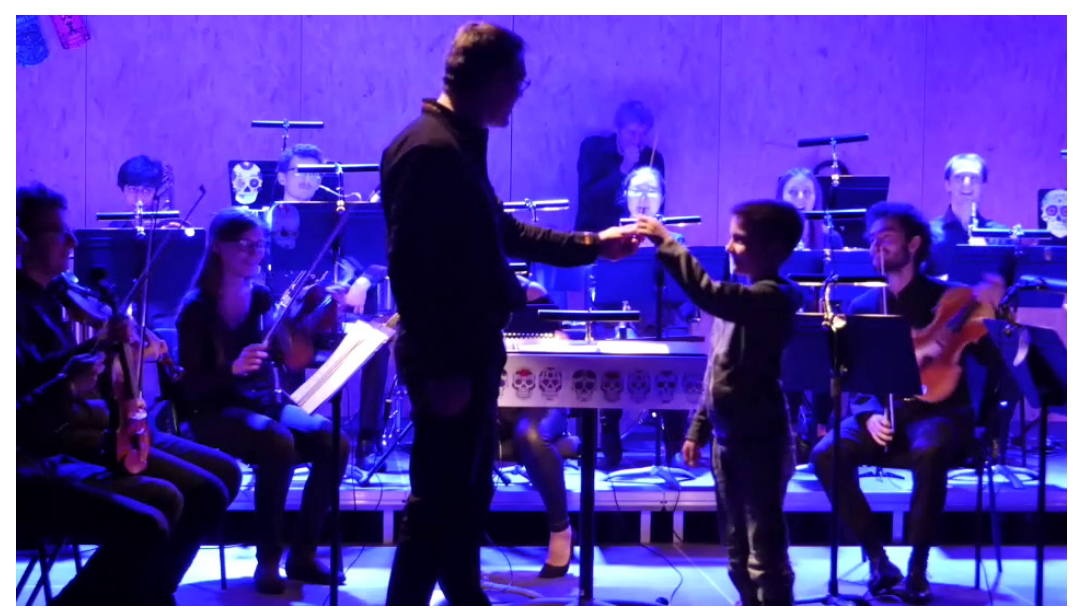

Figure 5 : L'enfant chef d'orchestre.

Cette activité singulière contribue au processus d'autonomisation professionnelle des jeunes musicien·ne·s, à leur prise de conscience de la diversité des rôles qu'ils elles peuvent endosser (interprète, médiateur, pédagogue, etc.) et facilite pour chacun·e d'entre eux·elles l'expression de soi dans un cadre privilégié de socialisation musicale (petite formation d'une quinzaine de musicien·ne·s) comme cela a pu être également observé au sein de la formation à la médiation proposée par l'Orchestre Français des Jeunes (Prévost-Thomas et Vessely 2014).

\section{UNE EXPÉRIENCE IMMERSIVE PLURIELLE}

Enfin, pour chaque acteur et actrice de ce dispositif, enfants spect-acteurs ou musicien·ne's-médiateur-rice's, chef d'orchestre ou artiste invité $\cdot$, la participation permet de vivre une réelle expérience immersive au contact d'autrui. Et dans le cas présent, la dimension participative est encouragée par des propositions pédagogiques et esthétiques qui renforcent l'aspect immersif.

Ainsi, tel que présenté en amont dans le dossier pédagogique, dossier de préparation au concert destiné aux enfants, parents et enseignant $\cdot e \cdot s$, qui a pour « ambition de proposer une appropriation par divers angles d'approche, tout en offrant de nombreux éléments de mise en œuvre visant le renforcement de l'expérience artistique et la participation interactive " (HEMU 2017b, p. 6), l'aspect purement participatif de ce projet artistique singulier mettait l'accent sur les actions et activités suivantes :

Guidés par un narrateur, petits et grands seront plongés, le temps d'un concert, dans l'ambiance claire-obscure du " Día de los muertos ", où les morts se mêlent aux vivants dans le tourbillon d'une danse effrénée...[mais 1]'expérience ne serait pas complète sans la participation des enfants au spectacle. Parés de leurs plus belles réalisations (masques [figure 6] et bracelets ${ }^{13}$ ), ceux-ci seront invités à suivre le comédien dans une procession inspirée de la culture mexicaine. (HEMU 2017a, p. 15)

13 Le dossier pédagogique de La nuit des Mayas propose des tutoriels de bracelets magiques à confectionner et de masques de cavaleras à colorier (mais aussi de maracas minute et des güiros à gratter). 


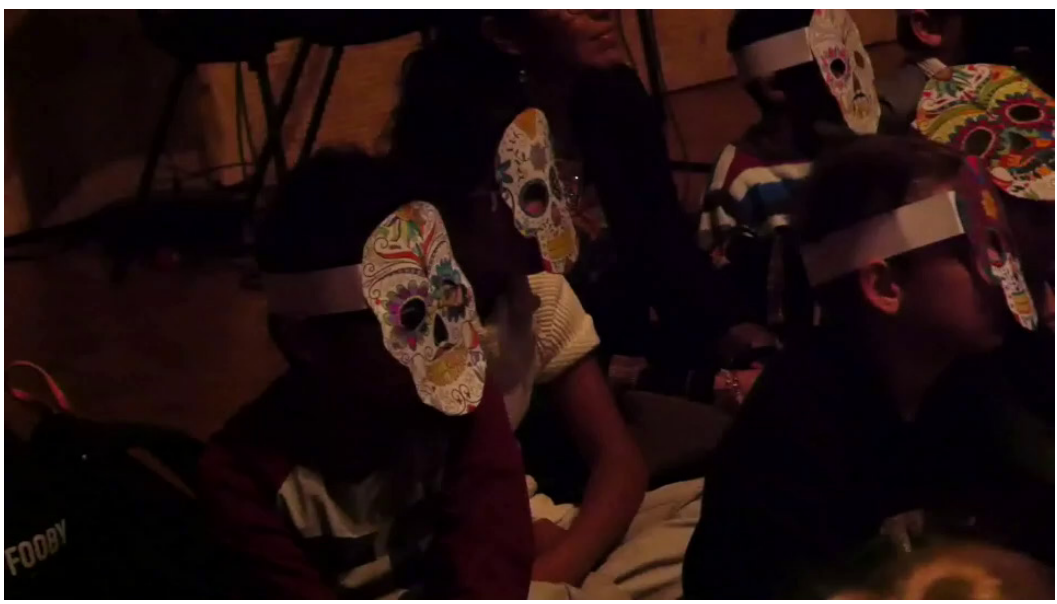

Figure 6 : Les enfants portent les masques de cavaleras qu'ils ont confectionné eux-mêmes.

Une fois l'œuvre de médiation musicale interprétée - par l'assemblage de Sensemayá de Silvestre Revueltas, de Danzón no 2 d'Arturo Márquez, du jeu des musicien'ne's et des interventions verbales du comédien et du chef d'orchestre -, la procession en musique s'est déroulée sous forme de déambulation au cœur même de l'orchestre (figure 7), au rythme d'un mouvement lent joué par l'ensemble des instruments présents, partie de l'œuvre délibérément choisie pour que les enfants profitent pleinement de l'instant, pouvant se mouvoir et vivre une expérience sensorielle au plus près des sons, des instruments et des musicien·ne·s, directement sur scène.

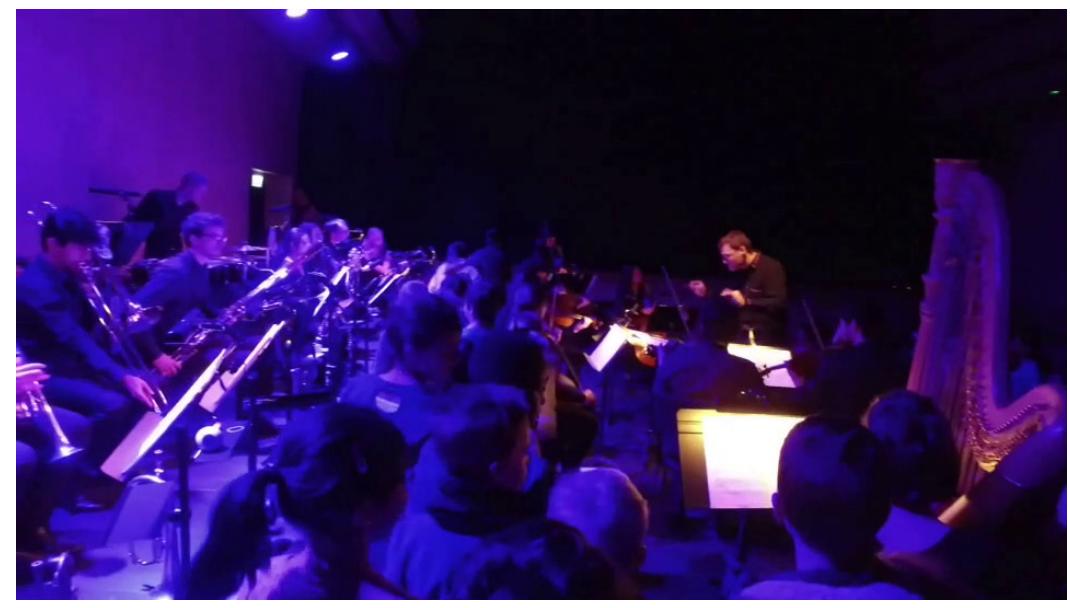

Figure 7 : Les enfants déambulent au cour de l'orchestre.

La dimension immersive agit ainsi sur la modification des rapports entre scène et salle. Opter pour une forme participative, c'est ainsi préférer la proximité à la distance, qui éloigne ordinairement les artistes du public et réciproquement. D'ailleurs, pour chaque concert-médiation de Musiques entre les lignes, quel que soit la forme choisie, le propos artistique privilégié, Weber repense la configuration de la salle au sein de laquelle va se dérouler la rencontre entre musicien'ne·s et publics. Dans le cas de "La nuit des Mayas », l'espace convenu habituellement a été adapté (figure 8). Ainsi, le rideau en fond de scène derrière lequel se place habituellement l'orchestre dans une relation verticale, a été délaissé 
pour une proposition spatiale horizontale où musicien'ne·s et publics se retrouvent dans la partie de la salle ordinairement réservée au public.

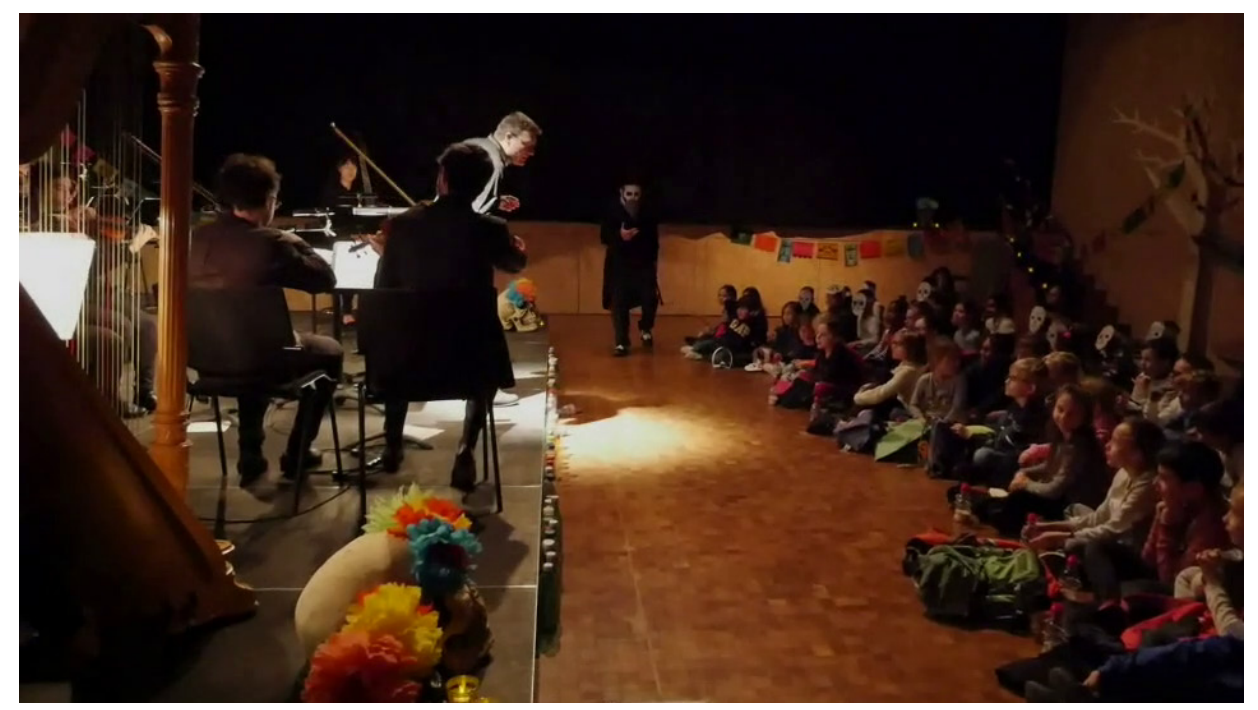

Figure 8: Un espace de médiation adapté.

De plus, la scène agencée sur des estrades de hauteur minimale surplombe à peine le niveau du sol où sont assis directement les enfants, plus libres de leurs mouvements et plus à l'aise, plus décontractés que dans une assise de salle de concert classique. Ces choix sont également effectués pour rapprocher physiquement la musique du public et donc accentuer la dimension participative du concert en privilégiant le caractère intime de la rencontre.

Enfin, l'alternance d'éclairages sombres et lumineux, de couleurs froides et chaudes, souligne l'intrigue musicale (figure 9), renforce l'adhésion au propos et soutient l'aspect immersif du dispositif qui encourage la participation de chacun·e.

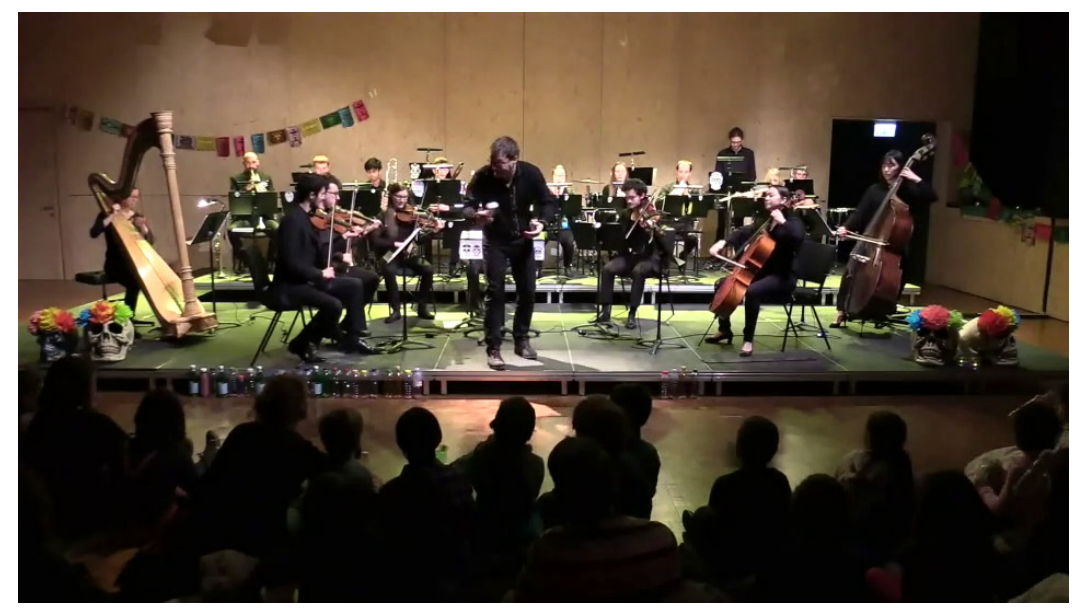

Figure 9: Des éclairages pensés pour la médiation. 
LES CATÉGORIES DE PARTICIPATION : DE L'INTERACTION À LA COCRÉATION

La présentation de la conjonction de ces différentes dimensions interactionnelles, esthétiques et symboliques incluses au sein du concert participatif " La nuit des Mayas », qui nous a permis de mesurer l'efficience d'un tel dispositif à la médiation de la musique classique, nous invite à un retour théorique sur les niveaux, degrés ou catégories de participation que l'on peut observer plus largement aujourd'hui dans les dispositifs dits d'art participatif afin de comprendre dans quelle perspective se situe la proposition de l'HEMU. Deux publications récentes se sont intéressées à ces questions de modalités participatives dans le champ artistique et culturel.

L'article "Art participatif et médiation culturelle. Typologie et enjeux des pratiques " de Nathalie Casemajor, Ève Lamoureux, Danièle Racine, déjà cité, propose une analyse à plusieurs entrées qui distingue les catégories "interaction ", " réappropriation ", " collaboration » et " cocréation " ${ }^{14}$. Si l'on soumet notre terrain à leur grille d'analyse, la première catégorie nommée " interaction » semble à première vue la mieux correspondre puisqu'elle «implique un type de participation caractérisé par une auctorialité forte de l'artiste professionnel ", qui autorise une interaction " avec le dispositif en place, selon un "mode d'emploi" défini par l'artiste » [...], » qui maîtrise seul la création du concept artistique et reste clairement l'auteur, le signataire de l'œuvre » (Casemajor, Lamoureux et Racine 2015, p. 176). Comme tout programme de Musiques entre les lignes, "La nuit des Mayas » est en effet d'abord une création qui se déploie selon la forme du « concert » exposant des œuvres, des musicien'ne's, un orchestre et un chef, puisque l'une des ambitions du programme est de transmettre les codes du concert classique au jeune public.

Si les catégories réappropriation et cocréation semblent éloignées du dispositif observé ici, en revanche la médiation inhérente au programme Musiques entre les lignes correspond pleinement à la troisième catégorie de participation proposée par les auteures, soit celle de la " collaboration ". Dans cette catégorie en effet, " les participants jouent un rôle de collaborateurs dans une proposition artistique qui émane de l'artiste, mais à laquelle ils peuvent contribuer. L'artiste y joue, en quelque sorte, un rôle de directeur artistique. Il définit un cadre à l'intérieur duquel les participants vont inscrire leur contribution et nourrir le projet par un apport de thèmes, de sens, de créativité, et ce, en amont même de la production » (ibid., p. 178).

Si la marge de manœuvre des musicien·ne·s, en formation - à la médiation, à la prestation scénique, à la découverte de nouveaux univers musicaux, au contact de l'autre, au public, etc. - et des enfants, en apprentissage - de la musique classique, des codes du concert, des sons, des rythmes, des instruments, etc. - est plus faible que celle de musicien·ne·s professionnel·le·s aguerri·e·s à la médiation et d'un public plus averti pour créer les conditions optimales d'une collaboration « en amont même de la production », la main tendue du chef-médiateur qui crée du lien avec les musicien·ne·s

14 Leur grille d'analyse schématisée sous forme de tableau apparaît à la p. 173 de l'article et à la p. 3 de la reproduction du texte disponible ici. 
(les associer au projet, leur faire découvrir des œuvres, les inviter à découvrir des univers artistiques différents du leur, leur transmettre le goût de s'adresser directement au public, d'improviser avec lui, de répondre à ses attentes, etc.) et avec les publics (leur faire découvrir des langages musicaux, les inviter à s'exprimer verbalement, physiquement, rebondir sur leurs réactions spontanées, les guider sur le chemin de l'émancipation, etc.), sont autant d'actions observées qui encouragent le développement toujours plus grand de cette dimension participative collaborative qui contribue sans nul doute au succès de ce dispositif de médiation musicale.

\section{VERS UNE MÉDIATION COLLABORATIVE}

Afin de mesurer pleinement l'équilibre du concert-médiation dans sa dimension participative, entre interaction et collaboration, une seconde lecture, celle de l'article de Chaumier, "Vers une médiation participative ", propose de distinguer médiation participative, médiation contributive et médiation collaborative. L'argument de l'auteur nous invite à poursuivre notre réflexion puisqu'il nous dit que si la médiation participative est agréable et la médiation contributive utile, la médiation collaborative, est, elle, indispensable : "Il ne s'agit pas alors des proposer des actions dans lesquelles les publics viennent s'inscrire, mais de favoriser une collaboration dès le début pour construire et envisager ensemble une action "(Chaumier 2016, p. 241). Si cette médiation a été expérimentée une première fois à l'HEMU en mars 2018 avec les musicien·ne·s en formation pour le concert-médiation Planet Music encadré par Jérôme Thiébaux ${ }^{15}$, elle reste à imaginer du côté des publics sans bien sûr dénaturer l'intention artistique initiale.

Associer à un tel projet participatif un·e médiateur-rice de la musique, qui ne serait ni artiste ni public mais suffisamment connaisseur euse de l'ensemble des enjeux qui les relie, pourrait permettre de tisser plus fortement les liens entre chaque partie et enrichir plus encore ce type de dispositif en prolongeant le temps de la médiation en amont et en aval dans les classes, par un échange épistolaire avec les familles ou par un temps d'échange prolongé avant et après les spectacles pour que la rencontre entre publics et musicien·ne·s soit encore plus opérante.

Une telle association pourrait résoudre l'équation d'une meilleure connaissance et appropriation des œuvres proposées avec l'expérience esthétique et relationnelle du concert-médiation. Si la médiation fait étymologiquement référence au milieu, l'approche de Musiques entre les lignes trouve son efficience à ce point d'équilibre : transmettre des contenus artistiques et culturels sans les imposer et autoriser la participation, le partage et la rencontre le temps de l'expérience du concert. Ainsi, « la médiation prend la place qui est la sienne à savoir le centre du motif à partir duquel les actions doivent être pensées et conduites » (ibid., p. 243).

Adhérer à cette catégorie de médiation «suppose d'affirmer et de reconnaitre que la finalité de l'action culturelle n'est pas dans le résultat, le spectacle ou l'exposition,

15 Jérôme Thiébaux a été professeur de médiation de la musique chargé des enseignements pratiques à 1'HEMU entre 2017 et 2019. 
et encore moins dans la sauvegarde du patrimoine pour lui-même, mais que celui-ci n'est qu'un outil au service d'autre chose, à savoir les hommes rassemblés dans une communauté de partage »(Chaumier 2016, p. 243).

Cette vision démocratique nous éloigne de celle imposant une sorte de " professionnalisation de la participation qui se concentre sur la communication et le marketing » (Carrel 2017, p. 29), où la médiation, souvent pensée sur un temps restreint ou comme activité périphérique, est davantage au service de l'image de l'institution culturelle (Prévost-Thomas 2016) qu'à celui de l'expérience esthétique efficiente partagée par ses usagers et ses spectateurs. Ce genre de proposition artistique nécessite donc d'approfondir une réflexion sur le temps global et qualitatif qui doit être spécifiquement accordé aux activités de médiation.

\section{Participation CUlturelle ou CUlture PARTicipative?}

L'expérience du concert participatif relatée ici nous invite enfin à poursuivre et conclure momentanément notre réflexion en revenant sur la notion centrale de " participation culturelle», prolongée par celle de « culture participative ».

Dans la dynamique de la Déclaration de Fribourg, selon l'Office fédéral de la culture (OFC),

le terme de participation culturelle désigne un objectif prioritaire de la politique culturelle. Il s'agit de donner au plus grand nombre possible de personnes - et ce en dépit de l'inégalité des chances au départ, inégalités touchant à la formation, aux revenus et à l'origine - accès à la culture, leur permettre de débattre de la culture et de pratiquer des activités culturelles. Renforcer la participation culturelle, c'est non seulement contrer les obstacles qui peuvent empêcher l'accès à l'offre et à la vie culturelle, mais c'est aussi soutenir les activités culturelles autonomes. Quiconque participe à la vie culturelle prend conscience de ses racines culturelles, développe une identité culturelle propre et contribue ainsi à la diversité culturelle de la Suisse. (

Le concept de " culture participative ", quant à lui, s'opposant à celui de culture consumériste, a été développé par le professeur Henri Jenkins et se définit ainsi :

Une culture participative est une culture conçue comme " ensemble de pratiques comportant des valeurs et des normes partagées » qui rapproche l'expression artistique de l'engagement civique, qui offre un fort soutien à la création et au partage de créations, et qui sous-tend une forme de mentorat informel entre les anciens et les nouveaux membres de cette culture. Une culture participative est également une culture dans laquelle les membres croient en la valeur de leurs contributions, et reconnaissent les liens sociaux forgés entre eux. (Jenkins 2009, p. 4)

Peut-être est-ce alors à l'intersection de ces deux visions que, grâce au cadre offert par l'activité de médiation, la participation encourage la conscientisation culturelle de chacun, qui elle-même favorise l'émancipation des acteurs, qu'ils soient musicien·ne's ou publics. En effet comme l'explique Chaumier :

C'est profondément une visée émancipatrice qui est contenue dans la dimension culturelle. Par elle, l'individu s'épanouit car il s'appartient davantage. Par l'art et la culture, il peut étendre la palette de ses émotions, ressentir et exprimer avec 
davantage de variations. La même chose peut être dite des sensations, des idées ou des mises en situation. En ce sens, on a pu dire que l'absence de culture est une perte ou une privation, une misère inconsciente d'elle-même. Celle dont on ne prend conscience qu'en la découvrant. (Chaumier 2016, p. 228)

De plus, la participation effective annonce pleinement le temps du partage :

C'est en découvrant l'Autre que l'on se découvre soi-même, c'est aussi en découvrant l'Autre que l'on découvre les autres. Première mise en relation et premier partage par le biais de l'artiste, mais aussi par le fait d'en partager l'expérience avec d'autres rassemblés pour l'occasion dans une communauté. [...] Ce qui se joue alors est la rencontre, dans un collectif rassemblé, d'une occasion de partage. (ibid., p. 229)

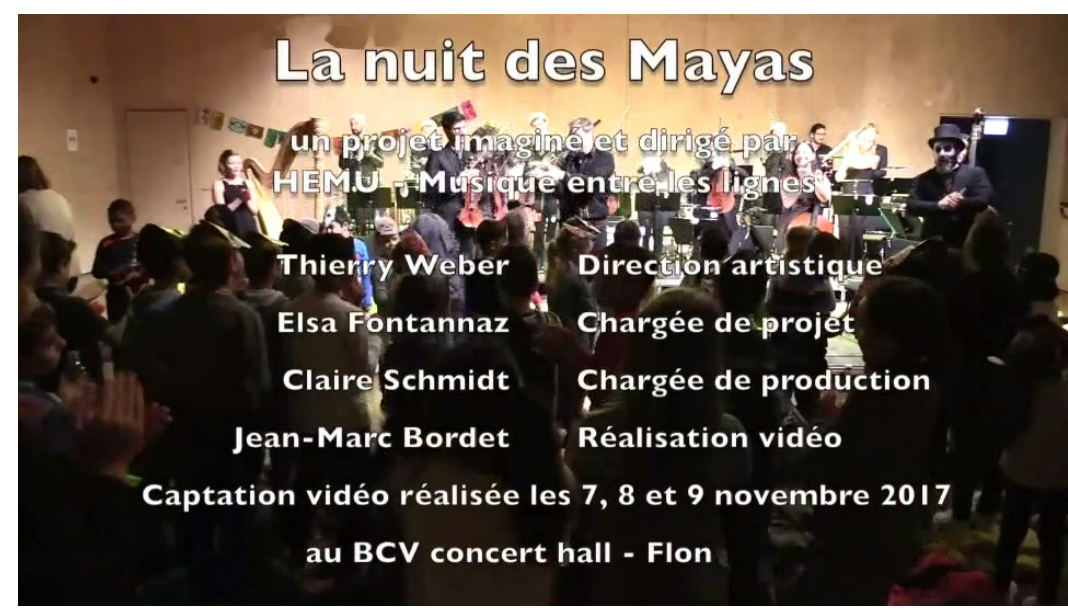

Extrait vidéo: Jean-Marc Bordet (réal.), La nuit des Mayas, captation vidéo réalisée les 7, 8 et 9 novembre 2017 au BVC Concert Hall, Flon, Lausanne, 4 min 38 s. Voir la vidéo.

\section{SUPPORTS DE MÉDIATION}

HEMU (2016), Musique entre les lignes - "On ne naît pas auditeur averti..., on le devient!", plaquette de présentation de la saison 2016-2017, 8 p.

HEMU (2017a), Musique entre les lignes, La nuit des Mayas - concert participatif, dossier pédagogique, Haute École de Musique de Lausanne, 22 p., https://www.hemu.ch/fileadmin/documents/pdf/ Agenda/MEL01Nuit des Mayas DP.pdf, consulté le 8 septembre 2020.

HEMU (2017b), Musique entre les lignes - "On ne naît pas auditeur averti..., on le devient! ", plaquette de présentation de la saison 2017-2018, 17 p.

\section{BiBLIOGRAPHIE}

Arnaud, Lionel(2015), «Action culturelle etémancipation par la culture. Un éclairage socio-historique», Informations sociales, vol. 190, $\mathrm{n}^{\circ}$ 4, p. 46-56, https://www.cairn.info/revue-informations-sociales2015-4-page-46.htm, consulté le 8 septembre 2020.

Binet, Jérôme (2016), " "L'important c'est de participer", reste à savoir comment », L'Observatoire, vol. 47, $\mathrm{n}^{\circ}$ 1, p. 76-79, https://www.cairn.info/revue-1-observatoire-2016-1-page-76.htm, consulté le 8 septembre 2020.

Boal, Augusto ([1977]1996), Théâtre de l'opprimé, Paris, La Découverte/Poche. 
Bocelli, Gianluigi (2017), «Créer un lien entre l'œuvre et le public », Schweizer Musikzeitung, n 6 (juin), p. 13-15.

Bourriaud, Nicolas (1998), Esthétique relationnelle, Dijon, Les Presses du Réel.

Bresson, Maryse (2014), « La participation : un concept constamment réinventé. Analyse sociologique des enjeux de son usage et de ses variations ", Socio-logos, $\mathrm{n}^{\circ}$ 9, http://journals.openedition.org/ socio-logos/2817, consulté le 8 septembre 2020.

Camart, Cécile, et al. (dir.) (2016), Les mondes de la médiation culturelle, 2 vol., Paris, L'Harmattan.

Carrel, Marion (2017), "Injonction participative ou empowerment ? Les enjeux de la participation », Vie sociale, vol. 19, no 3, p. 27-34, https://www.cairn.info/revue-vie-sociale-2017-3-page-27.htm, consulté le 8 septembre 2020.

Casemajor, Nathalie, Ève Lamoureux, et Danièle Racine (2015), "Art participatif et médiation culturelle. Typologie et enjeux des pratiques ", dans Camart et al. 2016, vol. 1 p. 171-184.

Caune, Jean (2017), La médiation culturelle. Expérience esthétique et construction du Vivre-ensemble, Grenoble, Presses Universitaires de Grenoble.

Chaumier, Serge (2016) «Vers une médiation participative», dans Fanny Serain et al. (dir.), La médiation culturelle. Cinquième roue du carrosse?, Paris, L'Harmattan, p. 223-247.

Coen, Pierre-François, et Thierry Weber (2017), Images de la médiation de la musique en Suisse romande. Passer d'une expérience artistique à des questionnements pédagogiques, document non publié, Lausanne, Institut romande de pédagogie musicale.

Dorin, Stéphane (dir.) (2018), Déchiffrer les publics de la musique classique. Perspectives comparatives, historiques et sociologiques, Paris, Édition des Archives contemporaines.

Dubasque, Didier (2017), « Participer, oui, mais comment ? Éloge de la co-construction », Vie sociale, vol. 19, no 3, p. 73-78, https://www.cairn.info/revue-vie-sociale-2017-3-page-73.htm, consulté le 8 septembre 2020 .

Fontannaz, Elsa, et Lise Gremion (2018), " "Musiques entre les lignes" déploie ses ailes ", Nuances. Le magazine de la Haute École de Musique et du Conservatoire de Lausanne, no 55 , p. 47, https:/ / www.hemu. ch/fileadmin/documents/pdf/Nuances/Nuances 55-bd.pdf, consulté le 8 septembre 2020.

Inghelbrecht, Désiré-Émile (1957), Le chef d'orchestre parle au public, Paris, Julliard.

Jenkins, Henry (2009), Confronting the Challenges of Participatory Culture. Media Education for the $21^{\text {st }}$ Century, Cambridge, MIT Press.

Kaine, Élisabeth, Olivier Bergeron-Martel, et Carl Morasse (2017), " L'artiste-médiateur. Un transmetteur de l'expérience de l'autre ", dans Nathalie Casemajor et al. (dir.), Expériences critiques de la médiation culturelle, Québec, PUL, p. 321-342.

Ladsous, Jacques (2017), « La participation. Cela s'apprend, cela se cultive », Vie sociale, vol. 19, $\mathrm{n}^{\circ}$ 3, p. 209-214, https://www.cairn.info/revue-vie-sociale-2017-3-page-209.htm, consulté le 8 septembre 2020 .

Lamoureux, Ève, et Magali Uhl (dir.) (2018), Le vivre-ensemble à l'épreuve des pratiques culturelles et artistiques contemporaines, Québec, PUL.

Les droits culturels. Déclaration de Fribourg, https://droitsculturels.org/ressources/wp-content/uploads/ sites/2/2012/07/DeclarationFribourg.pdf, consulté le 8 septembre 2020.

Office fédéral de la culture (2016), La politique culturelle de l'Office fédéral de la culture pendant la période 2016 à 2020 ; les nouveautés dans le domaine des recherches de provenance, https://www.newsd.admin. ch/newsd/message/attachments/42684.pdf, consulté le 8 septembre 2020.

Pailler, Danielle (2018), « La participation des habitants à la vie culturelle peut-elle se passer de médiation?", L'Observatoire, vol. 51, no 1, p. 61-63, https://www.cairn.info/revue-1-observatoire2018-1-page-61.htm, consulté le 8 septembre 2020.

Pailler, Danielle, et Caroline Urbain (dir.) (2016), Médiation et participation. Participation et médiation(s), nouveaux regards pour de nouveaux enjeux, Paris, L'Harmattan. 
Penven, Alain (2015), «Cuisines et dépendances de la participation», Vie sociale, vol. 97, n 1 , p. 141-146, https://www.cairn.info/revue-empan-2015-1-page-141.htm, consulté le 8 septembre 2020.

Prévost-Thomas, Cécile (2006), Dialectiques et fonctions symboliques de la chanson francophone contemporaine, thèse de doctorat en sociologie, Université Paris X-Nanterre.

Prévost-Thomas, Cécile (2016), «Culture participative, responsabilité artistique et politique-L'exemple des pratiques musicales collectives ", Communication au xx congrès de l'AISLF, "Sociétés en mouvement, sociologie en changement ", Montréal, 4-8 juillet.

Prévost-Thomas, Cécile, et al. (2015) « Table ronde. Médiations musicales : enjeux esthétiques, politiques et professionnels », dans Camart et al. 2015, p. 163-181.

Prévost-Thomas, Cécile, et Pauline Vessely (2014), « Du musicien médiateur au médiateur musicologue. Entre intégration sociale et socialisations musicales », dans Frédérique Montandon et Thérèse PérezRoux (dir.), Les médiations culturelles et artistiques. Quels processus d'intégration et de socialisation?, Paris, L'Harmattan, p. 57-76.

Ruby, Christian (2017), Devenir spectateur? Invention et mutation du public culturel, Toulouse, Éd. de l'Attribut.

Schütz, Alfred ([1971]1884), "Faire de la musique ensemble. Une étude des rapports sociaux », Extraits de "Making Music Together - A Study in Social Relationship », Collected papers II, La Haye, p. 159-178 ; Sociétés, vol. 1, no 1, p. 22-27 ; voir aussi https://www.cairn.info/revuesocietes-2006-3-page-15.htm, consulté le 21 septembre 2020.

Schütz, Alfred (2007), "Faire de la musique ensemble. Une étude de la relation sociale - 1951 », Écrits sur la musique, Paris, MF, p. 115-139.

Siohan, Robert (1967), Histoire du public musical, Lausanne, Les éditions Rencontre. 